Reflecting on the research encounter for people in the early stages of dementia: lessons from an embedded qualitative study

Michele Abendstern, PSSRU, University of Manchester

Karen Davies, PSSRU, University of Manchester

Fiona Poland, University of East Anglia

Helen Chester, PSSRU, University of Manchester

Paul Clarkson, PSSRU, University of Manchester

Jane Hughes, PSSRU, University of Manchester

Caroline Sutcliffe, PSSRU, University of Manchester

David Challis, PSSRU, University of Manchester 


\section{Reflecting on the research encounter for people in the early stages of dementia: lessons from an embedded qualitative study}

\section{Abstract}

Gathering meaningful data from people with dementia presents challenges to researchers involved in both qualitative and quantitative studies. Careful planning and implementation are required, including skilful and sympathetic management by the researcher who must pay attention to the cognitive challenges experienced by the person with dementia. These challenges are particularly evident when conducting structured interviews using standardised measures. This paper presents the findings of an embedded qualitative study undertaken within a pragmatic RCT. The novel method involves nesting a qualitative analysis within a quantitative study by recording incidental conversation during structured interviews, requiring no additional data collection. The method shone a light on the formal interview process itself, something rarely revealed outside the interview setting. It provided a unique insight into the challenges posed by research participation for people in early-stage dementia. Analysis revealed three main themes relating to dementia as a condition and to the research design. First, people with dementia contributed very few conversational comments during the structured interviews. Second, the context of the interview, that is: managing the conversational interchange, responding to direct and often sensitive questions and making decisions about day-to day-feelings and experiences was difficult for participants to manage. Third, people in early stage dementia struggled with the content of the structured interviews due to their linguistic and cognitive demands. The findings raise questions about how people with dementia are included in research and the methods employed to gather accurate data with minimal inconvenience and stress for research participants.

\section{Introduction}

Policy and practice has increasingly recognised the need both to understand the experiences of people with dementia and also ensure that they are involved in decision making about their own care (Digby, Lee \& Williams, 2016; Hubbard, Downs \& Tester, 2003; McKeown, 2017; Murphy et al., 2015). The voices and presence of people with dementia within research has frequently been marginalised (Hubbard et al., 2003; Hampson \& Morris 2018; Taylor et al., 2012; Hellstrom et al., 2007; Lepore, Shuman, \& Weiner 2017). However, there is now an expectation by the research community that studies relating to the condition will, where possible, directly involve people with dementia rather than relying on proxies 
speaking on their behalf (Keady et al., 2017). At the same time, it is acknowledged by researchers such as Keady that gathering data requires careful planning and implementation. Study design needs to be attentive to the cognitive challenges experienced by the person with dementia and the research process requires skilful and sympathetic management by the researcher interacting with the person with dementia and their family (Beadle-Brown et al., 2012; Digby, Lee \& Williams, 2016).

Research methods that are routinely used and validated for other populations, can pose difficulties for people with dementia (Phillipson \& Hammond, 2018). Qualitative research literature highlights three salient issues that need to be considered in undertaking research with people with dementia and which may have equal importance in the design and delivery of quantitative studies. First, if we are to hear the voice of the person with dementia clearly and accurately, innovative methods may need to be employed. Potentially, these can enable the accounts of individuals with limited communication and declining cognitive skills to be heard (Novek \& Wilkinson, 2017; Rivett, 2017). Qualitative researchers have drawn attention to the language used during data collection, pointing to the cognitive demands of questions as well as a tendency to focus on deficits on which people may be reluctant or unable to comment (Novek \& Wilkinson, 2017). Second, where structured interviews are used to collect data, the context may need to be adjusted to include family members or carers. Separating the person with dementia from their carer during a structured interview can be impractical, for instance if interviewing takes place within the carer's home (Morris, 2001). However, going on to include carers in research interview changes the dynamics to a three way interaction, altering the rules of engagement and presenting new challenges for the researcher and indeed the person with dementia, who may find a more complex conversational interchange difficult. Third, the researcher needs to be aware of the potential burden of research participation for the person with dementia. The content of research interviews, often using standardised measures, raises challenges in terms of complexity and length of questions and topics that deal with sensitive issues relating to health, wellbeing and social interaction. The ethical imperative to minimise the participant burden (Lingler et al, 2015) can create a tension when set against the obligation for researchers to maximise participation. Currently, there is limited evidence regarding the experience of people with dementia and their carers involved in quantitative studies (Taylor et al., 2012). However, one example, related to an RCT of immunotherapy (Solomon et al 2012), highlights a range of difficulties in encouraging full involvement of people with dementia and their carers. 
This paper considers the experience of people with dementia taking part in a pragmatic randomised control trial (RCT) investigating the effectiveness and cost-effectiveness of providing a range of memory aids, training and support to people with mild to moderate dementia and their carers at home (Chester et al., 2018). The trial forms part of a wider research program, Effective Home Support in Dementia Care: Components, Impacts and Costs of Tertiary Prevention, comprising nine interrelated projects funded through a National Institute for Health Research (NIHR) Program Grant for Applied Research. The current study used a novel embedded qualitative research design explicitly situated within a trial. The research aim was to extend our understanding of the experience of living with dementia, with a specific interest in changes in memory and mood, without increasing the burden on research participants. This entailed collecting naturally occurring qualitative data unobtrusively that had the potential to provide contextualised insights into interview topics and processes. The method afforded the opportunity to consider people's contributions during structured research interviews employing standardised measures, by analysing the interview process itself qualitatively, and observing both verbal comments and patterns of interchange between the person with dementia, their carer and interviewer. The method had been successfully employed with carers of people in later stage dementia, revealing rich data on lived experiences (Abendstern et al., 2018).

\section{Method}

The embedded qualitative study aimed to collect contextual and conversational data from participants during structured interviews for the main study. Evidence about participants' experiences and use of memory aids in combating memory loss was secured during audio recordings of their baseline interview.

\section{Participants and recruitment}

Participants for the RCT were recruited by researchers from NHS Trusts in England according to the inclusion criteria outlined in Box 1. Identification of mild to moderate (earlystage) dementia was based on assessment by their responsible clinician. The sample included people with a range of cognitive deficits/abilities. To be included in the main study, the person with dementia was required to have an identified carer, defined as the primary person who took responsibility for, and supported, them. This was broadly defined and could be a family member, a close friend or a neighbour. They did not need to live with their carer to take part in the study. 


\section{Box 1: Inclusion criteria for RCT}

1. Aged 50 years or more

2. Identified carer

3. Mild-moderate dementia identified by clinicians

4. Receiving support from memory clinic

5. Within one year of first attendance at clinic

6. Physically and clinically able to participate in a memory intervention programme

7. Live in their own home or share a home with a relative

Clinical staff within NHS Trusts introduced the study to participants and provided them with participant information sheets. They were given up to a week to decide whether or not they wished to take part in the RCT following receipt of the study information. At the baseline interview the study was explained again and formal informed consent was obtained using participant and carer consent forms. Interviewers were asked to judge whether the person with dementia had the capacity to provide informed consent to take part in the research. Where it was judged that they did not have such capacity the carer was asked to act as a consultee as defined within the Mental Capacity Act (2005). Participants were free to withdraw at any time without giving a reason.

In one participating Trust, a sub-group of those participating in the RCT were invited to have their interview audio-recorded as part of the embedded qualitative study so that their audiorecorded interviews could be analysed in detail. This sub-group was selected by asking all participants within a specified timeframe (January to August 2018) if they were willing to have their interview audio-recorded. There were no changes or adaptations to the interview for those that were audio recorded and interviewers were instructed to conduct these interviews in the same way as all others. This meant that every question on the interview schedule was asked of every participant. Techniques typically used in semi-structured qualitative interviews to actively elicit or clarify interviewees' meanings of experiences were not used as this was outside the remit of the structured interview method required by the $\mathrm{RCT}$. Interviewing was approached in an objective manner, accepting that there will always be an element of variation in social research involving people. The interview schedule is available as a supplementary document and reports the sequence of measures used. Research interviewers received online training about administering the standardised measures in a consistent and objective manner.

\section{Data collection}


The structured interviews contained a range of standardised measures administered by interviewers in participating NHS Trusts. These included measures such as the Standardised Mini-Mental State Examination (S-MMSE), Quality of Life measures and receipt of health and social care services (Box 2). Interviews were conducted face-to-face with the person with early-stage dementia and their carer. There were two interview schedules, one for the person with dementia and one for their carer. Respondents were given the flexibility to be interviewed either together or separately. People with dementia and their carers often expressed a preference for being interviewed together. Therefore, carers were often present during the interviews with people with dementia and any contribution they made necessarily formed part of the now three-way contextualising conversation analysed in the embedded qualitative study.

\section{Box 2: Measures completed by people with early stage dementia during structured interview for RCT}

Cognition

- The Standardised Mini-Mental State Examination (S-MMSE) measuring severity of cognitive symptoms of dementia ${ }^{1}$ (a)

Quality of life (three measures)

- Investigating Choice Experiments for the Preferences of Older People CAPability measure for Older people (ICECAP-O) measures quality of life in older people across five domains ${ }^{3}$ (a)

- The Control, Autonomy, Self-realisation and Pleasure 19-item measure (CASP-19), measures quality of life in older people across another four domains ${ }^{4}$

- The Dementia Quality of Life scale (DEMQOL) measures five domains of quality of life in dementia and has good validity and reliability. Completed by the person with dementia or the carer completes a proxy version

\section{Health-related quality of life}

- EQ-5D-5L provides a simple descriptive profile that generates a single utility value for health status to assess quality of life ${ }^{10}$

\section{Social network (two measures)}

- The Lubben Social Network Scale - Revised (LSNS-R) - designed to measure social isolation in older adults through perceived support from family and friends ${ }^{5}$

- The Practitioner Assessment of Network Type (PANT) allocates participants to one of five types of network reflecting their contact with family, friends and neighbours ${ }^{6}$

Resource use (two measures)

- The Client Service Receipt Inventory (CSRI) used extensively in studies of mental health and dementia to record details of formal services received ${ }^{7}$

- The Resource Utilisation in Dementia questionnaire (RUD). Estimates volume, duration and 
cost of support from formal and informal carers ${ }^{8}$

\section{Other data collected}

- Socio-demographic information and study specific measures including comorbidities in the person with dementia, current use of memory aids and current medication

(a) Completed by person with dementia (b) completed by person with dementia or carer

${ }^{1}$ Molloy and Standish (1997) ${ }^{2}$ Hughes et al (1982) ${ }^{3}$ Coast et al (2008) ${ }^{4}$ Hyde et al (2003) Netuveli et al (2006) ${ }^{5}$ Lubben et al $2003{ }^{6}$ Wenger (1991), ${ }^{7}$ Wenger and Tucker (2002) ${ }^{8}$ Beecham and Knapp (1992)

\section{Data analysis}

The analysis focused on both the additional comments that people with dementia made and on the interaction between the interviewer, carer and person with dementia. Interviews were transcribed verbatim and analysis of the data was managed using ATLASTi software. Two researchers conducted a thematic analysis using the six phase process of identifying themes and relationships across the data set (Braun et al., 2014). Initially the data was explored independently through a process of immersion in the entire data, followed by developing codes and searching for themes. Coding was inductive, using a process of systematically exploring participants' responses during the structured interview in order to identify meaningful themes. The researchers then developed a consensus for the codes and themes, together with a research advisory group. The themes were reviewed, checked and recoded where necessary, to ensure that the interpretation and final key concepts reflected the evidence accurately. The transcripts consisted of lengthy passages of questions followed by short responses with occasional detailed reflections from participants. The analysis involved a process of constant comparison across the whole interview to capture perspectives generated throughout the research encounter. As the analysis progressed, it became clear that there was not a consistent relationship between particular questions and measures and the type of comments that were generated. Therefore, the analysis moved beyond responses to particular questions and instead synthesised perspectives expressed throughout the interview into main themes and subthemes.

\section{Findings}

Fourteen dyads of people with dementia and their carers took part in the embedded qualitative study (Table 1), providing a total of twenty-eight participants. Interviews lasted an average of 1 hour 30 minutes (between $1 \mathrm{~h} 05 \mathrm{~m}-2 \mathrm{~h} 20 \mathrm{~m}$ ). Participants consisted of twelve spouse couples (eleven lived together) and two mother and daughter pairs (one of whom lived together). Eight people with dementia were female and six were male. The age range 
of participants was 58 years to 91 years (Table 1). S-MMSE scores revealed that the majority of the sample could be classified as people with mild dementia using this measure (scores of 20-24). There were also two with scores lower than 20 and three with scores higher than 24.

As the analysis progressed it emerged that the themes related less to participants' comments about their lived experience, in contrast to a previous study with carers (Abendstern et al., 2018). Participants provided relatively few additional comments about living with dementia. However, by reflecting on this in context it revealed a powerful commentary on the nature of involving individuals in research using standardised research measures, potentially identifying important issues for future research. The analysis yielded evidence of the challenges of participating in structured research interviews, both in the comments that participants made and in their reactions during the interviews.

\section{TABLE 1 PLACED HERE}

Analysis of the interviews revealed three main themes relating to the experience of the person with dementia, particularly as a research participant (Table 2). First, people with dementia contributed very few conversational comments during the structured interviews. Second, the context of the interview and third, its content were often, with some exceptions, seen to pose difficulties for participants to negotiate. These themes related to the features dementia as a condition and the nature of the research design and delivery. Findings are discussed below using illustrative quotations from the interviews. The participants are noted as 'l' for interviewer, ' $\mathrm{P}$ ' for participant and ' $\mathrm{C}$ ' for carer. Any names are indicated by initials in the quotations.

\section{TABLE 2 PLACED HERE}

\section{Limited conversational comments from people with dementia}

Interview participants focused on answering formal questions with few additional comments to explain or qualify their answers. For instance, there are just five occasions over a long interview where Participant 10 adds anything to her answers. One such example is where she responds to a question about whether she has felt distressed in the past week, to which she replied: 'Everybody's a bit distressed sometimes'. The interviewer then asked if this distress was related to her memory to which she says: 'When [I] don't do things right, you 
know, make a mess of it'. The interview then moves on. Occasional dialogues were found in other interviews where people with dementia expressed something of how they perceived themselves or thought they were seen by others, providing glimpses into the deeper experiences of living with dementia. For example, in a good natured rather than confrontational conversational exchange about the 'correct' answer to a question, Participant 9 declares to his partner that, 'What you think and what I think is different. You're at work all day, E.' Another example is more explicit about the difficulties encountered, 'I try and start to tell people something and then I get myself all in a muddle... with the words' (P11), directly expressing her frustration. Brief comments by some participants suggested that they were aware that they did not have the same position in the family as in the past. Participant 9, for example, states that his children no longer come to him for advice: 'They know now my state and...they won't be asking me for advice...'. Participant 6 expressed a similar view, but in this case he was contradicted by his wife who reassured him that he remained the person his children sought advice from:

I: If one of your relatives has an important decision to make how often would they talk to you about it?

P6: Um, they tend to, er, talk to, um, $P$.

C6: No they don't. No, we do the gabbing. You do the talking, the proper talking. It's different. No, they...they all talk. If they've got anything they all talk to you, and, er, tea and talk too much.

P6: [Laughs].

I: So, really always they would...they would involve you in...important...

P6: Yeah

Interview questions occasionally triggered brief narratives by participants highlighting aspects of their lives, such as a past occupation or interest, although no consistent pattern was found to suggest specific questions were more or less likely to elicit such responses. These comments were frequently repeated, suggesting a desire to communicate something of themselves, their relationships, and their identity, as illustrated by Participant 14:

I: How often do you see any of your children or relatives to speak to?

P14:Oh, well, K every, er, I don't know twice a month. She...K is, er...l...l used to be ... I used to be a runner...a marathon runner. Twenty marathons. I'm a...skier. I'm a mountaineer. We've...climbed in the Alps.

I: Right, I bet you miss all of that. 
P14: And I miss all that because l'm...I'm getting a little bit old. So K comes here and then off we go running in the forest....And I'm very, very slow. But I do it. With $K$

Such narratives could also be seen to provide a means of demonstrating emotions, in linking these to tangible experiences. For example:

I: What about depression?

P3: $\quad$ Not......no not really

C3: $\quad G e t$ fed up sometimes, don't you. But...no not really

P3: No...I mean, all my life l've had dogs, er, taken them for walks. I've...I enjoy gardening...

I: Yes. Yeah.

C3: Yeah, we miss the dogs

P3: $\quad$....er, that kind of thing.

Such glimpses of participants' lived experience tended to be singular individual expressions rather than examples of themes evident across participants. They referred to frustration over memory lapses, as seen above, to their perceived changing roles and status, and to anger at losing their independence. In essence, the structured interviews offered people with dementia little scope to embellish their responses. Some of the reasons for this are explored below.

\section{Impact of interview context on responses by people with dementia}

Carers were present and were involved to a greater or lesser extent in all of the interviews with people with dementia. The presence of an additional participant, often necessary for practical reasons but without a formal role in the interview, at times contributed to confused conversational interchanges. Carers varied in how frequently they intervened, some opted to remain largely silent, some to gently prompt. Others sought to encourage and support the person with dementia to respond accurately to the questions posed by the interviewer. However, there were also examples where the carer took a different, even more prominent role in the interview, typified by correcting or contradicting the participant:

l: $\quad$ have you felt full of energy in the last week? A lot, quite a bit, a little or not at all.

P13: Quite a bit

I: $\quad$ Quite a bit 
C13: Oh, rubbish. Excuse me, that is.... lie

P13: Not as good as it used to be [laugh]

Sometimes in such cases, the interviewer would then try to ask the question again, prompting the person with dementia to give the same response as the carer. The effect was to modify or diminish the answers given by the person with dementia, as illustrated by Participant 8:

P8: I'm not worried about being muddled, am I?...You think I do worry about things like that?

C8: $\quad$ You've got to answer the question, not me.

P8: I can tell by your face

I: $\quad$ Aw. What... do you think you feel....it doesn't worry you?

P8: $\quad$ Well I suppose it does slightly but I mean I'm not...I don't know really

Whilst in this example, the carer's intervention could be seen as facilitating the person with dementia to reflect on their initial response and therefore articulate their feelings more accurately, there were other examples where the interviewer was left with little choice but to accept the more dominant voice of the carer:

I: $\quad$ Do you feel satisfied with the way your life has turned out?

P7: Yes

C7: Well, yes and no, because you're comment every day is you wish you'd have gone...died at the same time as dad.

P7: $\quad$ Oh, I'm only talking like that.

C7: Yes, I know, but that's what you're thinking ... Basically you wish you'd died with dad, is that the truth?

P7: I'm not thinking that a lot, a lot, but...

C7: No, but you do.

I: But you do...when you're feeling at your lowest ebb, that's how you feel.

P7: $\quad$ That he's not here.

C7: Or why didn't I go first?

I: Yeah, yeah and I would say that is depression and low mood, more than just being fed up.

P7: $\quad \mathrm{Mm}, \mathrm{mm}$.

\section{Impact of interview content on responses by people with dementia}


The findings also indicated that some participants felt anxious during the interview for reasons of content rather than context. This was indicated in several ways including misunderstanding questions and showing uncertainty about how to reply, giving answers that they seemed to think the interviewer wanted, conveying feeling pressured to say the right thing, and forgetting things during the memory 'test', that they later remembered at the end of the interview, sometimes an hour later. Some participants expressed distress at the prospect of the interview itself, commenting that they were unsure about what to expect:

I: $\quad$ So in the last week, have you been worried about how you're feeling or...?

P1: I've been worried about this.

l: $\quad$ About coming?

P1: Yeah [laughs]

I: $\quad$ So would you say it was a little, quite a bit?

P1: A little

I: $\quad$ A little

P1: $\quad$ Because I didn't know what to expect [laugh]

There were examples of participants expressing confusion when questioned, evident explicitly or indicated through their uncertainty as they answered. On occasions the interviewer apologised for the difficulty of the questions and attempted to explain why questions were repeated, 'what they've actually done, I think, is they're all evidence based but they're taken from different things. So some of them.... are a little repetitive'. On other occasions the dialogue became very confused with interviewer, participants and carers talking over each other, attempting to clarify and reinterpret what was being asked. In the following example, the confusion was compounded by a second interviewer (I2) who was shadowing the main interviewer:

l: How often do you see or hear from the relative with whom you have the most contact? Who would you say that you have the most contact with? Is it one of your sons that... lives... The closest one, is it?

C2: $\quad$ The closest son, yeah

P2: Yeah.

I: How often do you see him, about once a week?

C2: Probably once a week... 


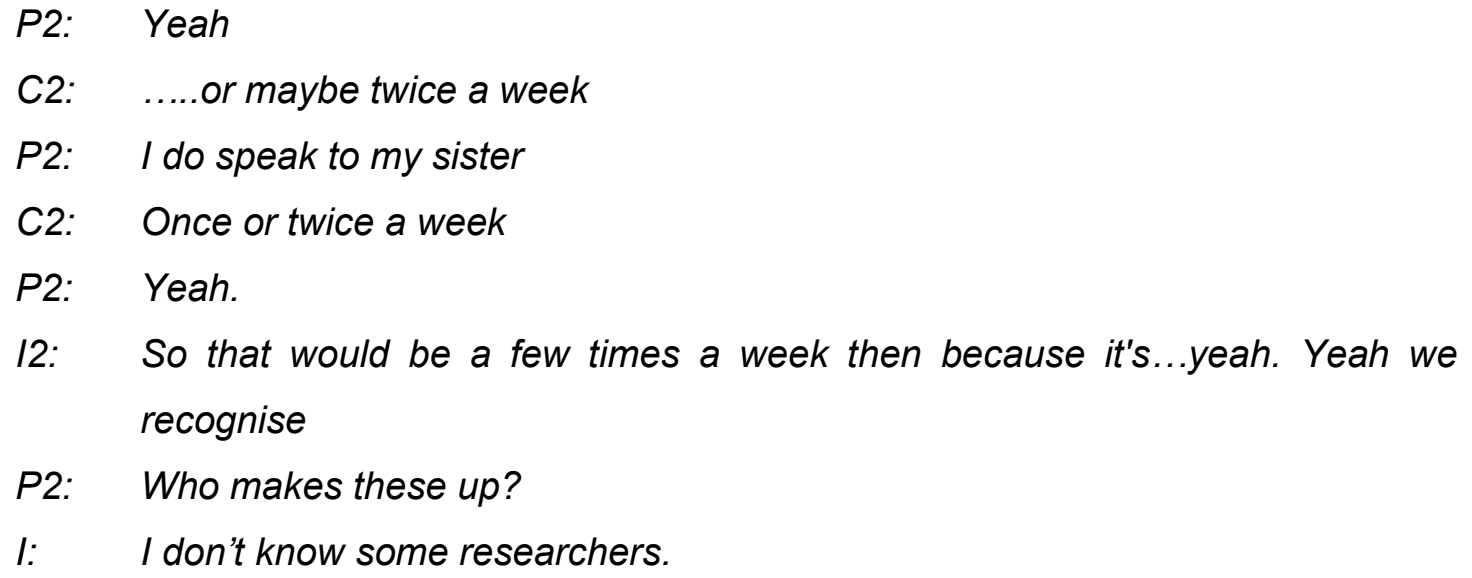

12: $\quad$ So that would be a few times a week then because it's...yeah. Yeah we recognise

P2: $\quad$ Who makes these up?

l: $\quad$ I don't know some researchers.

Many of the examples above illustrate how all three individuals in the interview are dealing with sensitive issues that could be distressing for the person with dementia and carer and difficult for the interviewer to manage. The interchange presented below, illustrates how the standardised questions are very direct in probing potentially emotionally difficult aspects of life, particularly in the context of older age and deteriorating cognition. In this example, the person with dementia shows initial resourcefulness in justifying his answer to the question about whether life is full of opportunities but appears to later capitulate when asked whether the future looks good to him:

I: Do you feel satisfied with the way your life has turned out?

P3: Yes, very satisfied.

I: Yeah. Um, do you feel that life is full of opportunities?

P3: What, for me now or for people in general?

I: It is...it's... like, now really. Yeah.

P3: Well, yes, so...

l: So sometimes or...?

P3: Um, sorry, for me personally?

I: Yes. Yeah.

P3: Um, yes...er, most things I want to do I still can do. I mean, um, I can still garden and walk around...and...

I: And what about the future? Do you feel that the future looks good to you?

P3: [Laugh]. I don't know how long the future's going to last, so [laugh].

I: Alright. Yeah.

P3: But, er, I'm happy at the moment

The presence of the carer in the interview may have been particularly problematic for both participant and carer, where the questions were personal, relating to the relationship 
between the couple, such as 'do you have all the love and friendship that you want?' A number of dyads responded to this with humour, but the potential for pressure to avoid answering such questions accurately is evident as here:

I: Getting the affection that you want, are you worried about that at all?

P2: $\quad$ Oh, well, there we are, $F$, there's a question.

C2: $\quad$ She doesn't get any.

I: [laugh].

P2: $\quad$ I get enough affection from my sons.

I: $\quad$ Ah.

l: $\quad$ [laugh].

P2: $\quad$ And the fish in the pond, if they've not been eaten by the heron

l: [laugh].

Interviewers showed that they were aware of the potential strain that the structured interview might place on participants due to its length and the nature of the questions, using phrases such as 'that's the end of your interrogation' (P11) or encouraging continued engagement by saying, 'we're nearly finished now' (P7), the latter in response to Participant 7 saying 'my gosh' when shown a list of response options for a new set of questions approximately one hour into what turned into a 90 minute interview.

\section{Discussion}

Using an embedded qualitative study method as part of a pragmatic RCT generated some interesting findings. The aim of the embedded qualitative study was to collect contextual and conversational data about participants' experiences of life in early stage dementia and their assessments of the role of memory aids in combating memory loss, with the intention of enhancing the understanding of the quantitative results. However, rather than providing a window on the wider lived experience of dementia, it shone a particular light on the formal interview process itself, something rarely conveyed or revealed outside the interview setting. It found that, in contrast to the extensive contextual discourse generated by carers identified in a previous embedded qualitative study (Abendstern et al., 2018), participants living with the early stages of dementia rarely added comments on their experience of living with dementia. Instead, the analysis of the interchanges between interviewer, carer and participant as they responded to questions from standardised measures, provided a unique insight into the specific challenges for people with mild to moderate dementia of being a research participant. This raises questions about the choices made regarding the means of 
gathering data which can appropriately tap and record the experiences of this participant group in future research. The remainder of the discussion considers what we can learn from these findings in order to ensure that future research maximises the opportunity to gather a range of data from people with dementia without compromising quality or imposing burden on participants.

\section{The influence of the research interview on the responses of the person with dementia}

These findings suggest that structured interviews include a significant memory and linguistic load that those who are newly diagnosed with dementia with mild to moderate dementia may find difficult to negotiate. Whilst structured interviews are not intended to encourage participants to comment and qualify their answers, recent research with carers has revealed how much rich data can be generated during such interviews that can enhance the findings of a study (Abendstern et al., 2018, under review). In contrast, data from the embedded qualitative study of people with dementia reported here revealed how rarely participants elaborated their answers. There were few examples of participants explicitly reflecting on what they wanted to say or attempting to think aloud to clarify or qualify their responses. These findings coincide with knowledge of early stage dementia, known to be characterised by cognitive impairment affecting communication, such as conversational skills, word finding and comprehension (Woodward, 2013). Whilst it could be argued that rich qualitative data, in the traditional sense, is therefore unlikely to be generated from this approach, the embedded qualitative method provided the opportunity to review the entire interchange between interviewer, carer and person with dementia across the duration of a structured interview. The richness of this data lay in the relationship between participants and the interaction between the person with dementia and the standardised measures. Structured interviews have an important place in assessing an individual's functioning. However, in the case of people with dementia, the findings of this study suggest that they may not always accurately reflect their experience or abilities. For instance, there are examples of carers 'correcting' answers given by people with dementia, suggesting that, at least in the eyes of the carer, their relative might overestimate what they could do. These findings suggest a need to collect qualitative data alongside the standardised measures to ensure the quality of the latter.

There is a growing interest in using innovative methods to overcome such limitations, such as observation techniques (e.g. Gibson et al., 2007) and the use of talking mats to help people with dementia to express their views (Murphy et al., 2010). Findings from the embedded qualitative study also support arguments for considering alternative ways of 
collecting essential information from people with dementia, such as proxy measures for factual information or using existing clinical data for MMSE scores. Such alternatives are not without their difficulties. Where we seek to understand people's experience, designs could also draw on qualitative methods such as storytelling (Osterholm \& Hyden, 2018). These encourage some people to narrate their experience, evident in some cases in this study. Alternative methods using visual adaptations of quality of life measures with people with severe mental health problems have been successfully trialled (Buitenweg et al., 2018) and may offer a more inclusive and accurate approach to assessing people with dementia in research studies. Examples of visual frameworks have been introduced in clinical practice to support decision making for people with dementia (Murphy \& Oliver, 2012) which could stimulate the design of alternative approaches for collecting research data directly from individuals with dementia (Naick et al., 2018).

\section{The influence of carers and interviewers on the responses of the person with dementia}

The findings also revealed how often people with dementia deferred to their care during the interview. Forbat and Henderson (2003) reported that interviewing people with dementia together with a carer created a new set of dynamics likely to change the responses made by the main participant to standardised measures. In the context of the real-world research observed in the current embedded qualitative study, such findings were also in evidence with people with dementia frequently influenced by others in the interview, both carers and interviewers. Other studies, however, have suggested benefits of dyadic interviews which have the potential to enrich the data (Bjornholt \& Farstad, 2014). For example, the 'cueing phenomenon' (Morgan \& Kruger, 1993), often evident in spouse's responses, may enable individuals to reveal more information; dyads can corroborate their joint story and potentially reveal more accurate detail of their experience, clarifying and modifying their accounts (Valentine, 1999). Examples of such features were evident in the findings from the current embedded qualitative study but were given little opportunity for elaboration given the closed nature of the questioning. Joint interviewing could improve the data collected through utilising interaction between individuals. However, to do this appropriately, preparation and training may be necessary to ensure that the perspective of the people with dementia can be genuinely and authentically prioritised.

There were several examples in this embedded qualitative study of interviewers touching on sensitive issues or of encountering difficult interchanges between people with dementia and their carer. Previous research has highlighted the tension that exists between systematic 
data collection and sympathetic and compassionate approaches to people participating in studies (Haahr et al., 2014; Orb, Eisenhauer \& Wynaden, 2001). Investigating participants' experience of the interview process, undertaken via the embedded qualitative research method in this study, highlighted three challenges relating to the study design, the relationship between researcher and participant, and the researcher's interpretation of responses, discussed in the field of qualitative research (Ramos, 1989). These issues are seen here to be equally pertinent to gathering data through structured interviews in quantitative studies. Some standardised measures gather data on people's experience of dementia (e.g. DEMQOL) and therefore may prompt sensitive disclosure by participants or be open to subjective interpretation by interviewers completing questionnaires, something more usually discussed in qualitative research. In studies such as this, where the person with dementia and carer are recruited and interviewed as a dyad, there is the added tension of negotiating the relationships between people with dementia, carers and the interviewer. Interviewers were seen to try to balance the need to be an objective researcher, eliciting accurate information from an individual who may have cognitive and communication difficulties, whist also maintaining a warm and supportive relationship with participants. The qualitative researcher is routinely urged to actively include opportunities to reflect on and review the research process. These findings from the embedded qualitative study suggest that this could also be an imperative for quantitative studies that use standardised measures. Considering the interactional demands, such as the number, complexity and form of questioning, could encourage researchers to consider alternative approaches that may reduce participant burden and develop 'dementia friendly' research that is sensitive as well as insightful. As this study has demonstrated, the addition of embedded qualitative research as part of large quantitative studies has the potential to encourage ongoing review of the research processes employed with people with dementia.

\section{Conclusion}

The embedded qualitative study provides an example of how generating and integrating qualitative and quantitative methods can highlight and provide more contextualised and focused understanding of research topics and the research design issues they raise. Attending to the interactional consequences of engaging people with dementia in research, including quantitative methods, enables researchers to reflect on the appropriateness of research design, and challenging preconceptions and assumptions about the benefits of specific approaches routinely used research. In exploring and extending the use of an innovative embedded qualitative research method, we have also generated novel findings 
and afforded a mechanism for reflecting constructively on interactive and experiential aspects of the research process. Such opportunities are not usually available in studies using quantitative methods. The embedded qualitative study provided critical insights into the merits and challenges of research methods and tools. This study bridges a notable gap in existing literature between using findings from qualitative studies about ways of appropriately engaging and recording the 'voice' of people with dementia and also realising the need to use standardised measures to provide consistency.

\section{Declarations}

Approvals: Ethical approval for the RCT was obtained from the National Research Ethics Service (NRES) (16/NW/0389).

\section{Consent for publication}

Not applicable.

\section{Availability of data and materials}

Not yet applicable.

\section{Competing interests}

The authors declare that they have no competing interests.

\section{Funding}

This project was funded by the National Institute for Health Research (NIHR) under its Program Grants for Applied Research (Grant reference number: DTC-RP-PG-0311-12003). The views expressed are those of the authors and not necessarily those of the NIHR or the Department of Health and Social Care.

\section{Acknowledgements:}

Authors' contributions:

\section{References}


Abendstern, M., Davies, K., Chester, H., Clarkson, P., Hughes, J., Sutcliffe, C., Poland, F. \& Challis, D. (2018). An embedded qualitative study of the views of family carers supporting people in the later stages of dementia. BMJ Geriatrics (under review).

Beadle-Brown, J., Ryan, S., Windle, K., Holder, J., Turnpenny, A., Smith, N., Richardson, L. \& Whelton, B. (2012). Engagement of people with long term conditions in health and social care research: Barriers and facilitators to capturing the views of seldom-heard populations, DP2850. Quality and Outcomes Research Unit, University of Kent, University of Oxford and LSE. Canterbury, UK: Personal Social Services Research Unit, University of Kent.

Beecham J. \& Knapp M. (1992). Costing psychiatric interventions. In G. Thornicroft, C. R., Brewin \& J. Wing (eds.), Measuring Mental Health Needs. London, UK: Gaskell.

Bjørnholt, M. \& Farstad, G.R., (2014). 'Am I rambling?' On the advantages of interviewing couples together. Qualitative Research, 14(1), 3-19.

Braun, V., Clarke, V. \& Terry, G. (2014). Thematic analysis. In P. Rohleder \& A. Lyons (eds.), Qualitative Research in Clinical Health Psychology (pp.95-114). New York, NY: Palgrave Macmillan.

Buitenweg, D.C., Bongers, I.L., van de Mheen, D., van Oers, H.A. \& Van Nieuwenhuizen, C. (2018). Worth a thousand words? Visual concept mapping of the quality of life of people with severe mental health problems. International Journal of Methods in Psychiatric Research, p.e1721.

Chester, H., Clarkson, P., Davies, L., Hughes, J., Saiful Islam, M., Kapur, N., Orrell, M., Peconi, J., Pitts, R., Poland, F., Russell, I., Challis D. \& Members of the HoSt-D (Home Support in Dementia) Programme Management Group (forthcoming) Cognitive aids for people with early stage dementia versus treatment as usual (Dementia Early Stage Cognitive Aids New Trial [DESCANT]): Study protocol for a randomised controlled trial. TRIALS.

Coast J., Flynn T. N., Natarajan, L., Sproston, K., Lewis, J., Louviere, J.J. \& Peters, T.J. (2008). Valuing the ICECAP capability index for older people. Social Science \& Medicine, $67,874-82$. 
Digby, R., Lee, S. \& Williams, A., (2016). Interviewing people with dementia in hospital: recommendations for researchers. Journal of Clinical Nursing, 25(7-8), 1156-1165.

EuroQol Research Foundation. (2015). EQ-5D-5L user guide. Rotterdam: EQRF. https://eurogol.org/wp-content/uploads/2016/09/EQ-5D-5L UserGuide 2015.pdf. Accessed 03 August 2015.

Forbat, L. \& Henderson, J. (2003). "Stuck in the Middle with You": The Ethics and Process of Qualitative Research th Two People in an Intimate Relationship. Qualitative Health Research, 13(10), 1453-1462.

Gibson, G., Chalfont, G. E., Clarke, P.D., Torrington, J. M. \& Sixsmith, A.J. (2007). Housing and Connection to Nature for People with Dementia, Journal of Housing for the Elderly, 21(1-2), 55-72.

Haahr, A., Norlyk, A. \& Hall, E.O. (2014). Ethical challenges embedded in qualitative research interviews with close relatives. Nursing Ethics, 21(1), 6-15.

Hampson, C. \& Morris, K., (2018). Research into the experience of dementia: methodological and ethical challenges. Journal of Social Sciences \& Humanities, 1(1), 1519.

Hellström, I., Nolan, M., Nordenfelt, L. \& Lundh, U. (2007). Ethical and methodological issues in interviewing persons with dementia. Nursing Ethics, 14(5), 608-619.

Hughes, C.P., Berg, L., Danziger, W. L., Coben, L. A. \& Martin, R. L. (1982). A new clinical scale for the staging of dementia. British Journal of Psychiatry,140, 566-72.

Hyde, M., Wiggins, R. D., Higgs, P. \& Blane, D. B. (2003). A measure of quality of life in early old age: the theory, development and properties of a needs satisfaction model (CASP19). Aging \& Mental Health, 7, 186-94.

Keady, J., Hydén, L.C., Johnson, A. \& Swarbrick, C. (eds.), (2017). Social Research Methods in Dementia Studies: Inclusion and Innovation. London, UK: Routledge.

Lepore, M., Shuman, S.B. \& Wiener, J.M. (2017). Challenges in Involving People with Dementia as Study Participants in Research on Care and Services, Background paper. 
Research Summit on Dementia Care. Building evidence for services and supports. US: Department of Health and Human Services.

Lingler, J.H., Schmidt, K.L., Gentry, A.L., Hu, L. \& Terhorst, L.A. (2014). A new measure of research participant burden: Brief report. Journal of Empirical Research on Human Research Ethics, 9(4), 46-49.

Lubben, J. \& Gironda M. (2003). Centrality of social ties to the health and well-being of older adults. In B, Berkman \& L. Harooytan (eds.), Social work and health care in an aging world (pp. 319-350). New York, NY: Springer

McKeown, J., Clarke, A., Ingleton, C. \& Repper, J. (2010). Actively involving people with dementia in qualitative research. Journal of Clinical Nursing, 19(13-14), 1935-1943.

McKeown J. (2017). Researching with People with Dementia. In: P. Liamputtong (ed.), Handbook of Research Methods in Health Social Sciences (1-15). Singapore: Springer.

Molloy D. \& Standish T. (1997). Mental status and neuropsychological assessment: a guide to the Standardised Mini-Mental State Examination. International Psychogeriatrics, 9, 87-94.

Morgan, D.L. \& Kruger, R. A. (1993). When to Use Focus Group and Why. In: D.L. Morgan (ed.), Successful Focus Groups: Advancing the State of the Art (pp. 3-19). Thousand Oaks, California, US: Sage publications.

Morris, S. M. (2001). Joint and individual interviewing in the context of cancer. Qualitative Health Research, 11(4), 553-567.

Murphy, J., Gray, C.M., van Achterberg, T., Wyke, S. \& Cox, S. (2010). The effectiveness of the Talking Mats framework in helping people with dementia to express their views on wellbeing. Dementia, 9(4), 454-472.

Murphy, K., Jordan, F., Hunter, A., Cooney, A. \& Casey, D. (2015). Articulating the strategies for maximising the inclusion of people with dementia in qualitative research studies. Dementia, 14(6), 800-824. 
Murphy, J. \& Oliver, T. (2013). The use of Talking Mats to support people with dementia and their carers to make decisions together. Health \& Social Care in the Community, 21(2), 171180

Naick, M., Towers, A-M., Smith, N., Palmer, S. \& Colloins, G. (2018). Developing and testing a Talking Mats symbol set to support conversations about social care-related quality of life with care home residents. British Society of Gerontology $47^{\text {th }}$ Annual Conference. Manchester, UK: British Society of Gerontology.

Netuveli, G., Wiggins, R., Hildon, Z., Montgomery, S. \& Blane, D. (2006). Quality of life at older ages: evidence from the English longitudinal study of aging (wave 1). Journal of Epidemiology andl Community Health, 60, 357-63.

Novek, S. \& Wilkinson, H. (2017). Safe and inclusive research practices for qualitative research involving people with dementia: a review of key issues and strategies. Dementia, 147. 1301217701274.

Orb, A., Eisenhauer, L. \& Wynaden, D. (2001). Ethics in qualitative research. Journal of Nursing Scholarship, 33(1), 93-96.

Österholm, J.H. \& Hydén, L.C. (2018). Autobiographical occasions in assessment meetings involving persons with dementia. Qualitative Social Work, 17(1), 41-64.

Phillipson, L. \& Hammond, A. (2018). More Than Talking: A Scoping Review of Innovative Approaches to Qualitative Research Involving People with Dementia. International Journal of Qualitative Methods, 17 [doi: 10.77/1609406918782784].

Ramos, M.C. (1989). Some ethical implications of qualitative research. Research in Nursing \& Health, 12(1), 57-63.

Rivett, E. (2017). Research involving people with dementia: A literature review. Working with Older People, 21(2), 107-114.

Smith, S.C., Lamping, D.L., Banerjee, S., Harwood, R., Foley, B., Smith, P., Cook, J.C., Murray, J., Prince, M., Levin, E., Mann, A. \& Knapp, M. (2005). Measurement of healthrelated quality of life for people with dementia: Development of a new instrument (DEMQOL) and an evaluation of current methodology. Health Technology Assessment, 9(10), 1-93. 
Solomon, A., Akenine, U., Andreasen, N., Mangialasche, F., Wimo, A., Jelic, V., Kivipelto, M. and Winblad, B., 2012. Practical lessons from amyloid immunotherapy trials in Alzheimer disease. Current Alzheimer Research, 9(10), pp.1126-1134.

Swarbrick, C. (2015). The quest for a new methodology for dementia care research. Dementia, 14(6) 713-715.

Taylor, J.S., DeMers, S.M., Vig, E.K. \& Borson, S. (2012). The disappearing subject: exclusion of people with cognitive impairment and dementia from geriatrics research. Journal of the American Geriatrics Society, 60(3), 413-419.

Valentine, G. (1999). Doing household research: interviewing couples together and apart. Area, 31(1),67-74.

Wenger, G. C. (1991). A network typology: from theory to practice. Journal of Aging Studies, $5,147-62$.

Wenger, G. \& Tucker, I. (2002). Using network variation in practice: identification of support network type. Health \& Social Care in the Community, 10(1), 28-35.

Woodward, M. (2013). Aspects of communication in Alzheimer's disease: clinical features and treatment options. International Psychogeriatrics, 25(6), 877-885. 